\title{
Crisis del paradigma organizativo-gerencial de la educación en Venezuela
}

Evaristo Méndez Quintero*

\section{Resumen}

Sostenemos que la actual crisis de la educación en Venezuela se explica fundamentalmente por el agotamiento del paradigma organizativo que le sirve de sustentación. Dicha crisis es la expresión final de una forma de organización social vinculada al proceso histórico denominado modernidad. La metodología utilizada fue holistica-causal al tener como finalidad comprender y explicar la crisis educativa a través del curriculum, la praxis educativa, el estilo gerencial-administrativo y la burocracia, determinando en cada caso los principios filosóficos y politicos del modelo de organización social, que impone la ciencia como única forma de entender la vida y la educación, acompañada de todo un mecanismo de control para garantizar la reproducción de ese modelo. Se concluye que la crisis se superará definiendo nuevos valores y prácticas docentes que asuman la ciencia y otras manifestaciones culturales como procesos abiertos y creativos que permitan alcanzar una calidad educativa y de vida en los estudiantes, profesores, directivos y comunidad en general.

Palabras claves: Educación, Burocracia, Gerencia, Modelo Organizativo y Venezuela.

\section{Crisis of the Organizative Management Paradigm Education in Venezuela}

\section{Abstract}

The main conclusion of this paper is that the current crisis of education in Venezuela is explained fundamentally by the exhaustion of the organizative paradigm on which it is

Recibldo: 21-03-96. Aceptado: 17-06-96

- Sociologo. Investigador del Centro de Estudios de la Empresa de la Facultad de Clencias Económicas y Soclales de la Universidad del Zulla. Profesor Titular. 
based. This crisis is the final expression of a social organization related to the historical process called modernity. The methodology applied was holistic - causal with the objetive both to explain and to understand the educative crisis throught the following elements. curriculum, educational praxis, administrative style and the bureaucracy. It is determined in each case the philosophical political principles of the social organizative model, that establishes science as the only way to understand life and education. Besides, it presents a control mechanism to guarantee the reproduction of this model. The conclusion is the crisis will be overcome by defineing both new values and teaching practices, it is assuming the science and other cultural expressions as open-creative process, to allow both an educative and life quality for studens, teachers, directors and community in general.

Key words: Education, Academic management, Bureaucracy, Curriculum, Venezuela

\section{Introducción}

La actual crisis de la Educación en Venezuela se explica fundamentalmente por el agotamiento del paradigma organizativo que la sustenta. Dicha crisis es la expresión final de una forma de organización social vinculada al proceso histórico denominado Modernidad. No es por lo tanto una crisis aislada del resto de la sociedad. Es la crisis misma manifestada en el fenómeno y proceso educativo. Para explicar este problema tenemos que ir a la esencia del proceso social y no quedarnos en la mera exposición de los acontecimientos que diariamente ocurren en el sistema educativo.

Este trabajo de análisis e interpretación socio-gerencial pretende explicar tanto la fenomenología como la esencia del proceso educativo en el contexto de la sociedad moderna y en particular la venezolana. En tal sentido, se ha diseñado una metodología holistico-causal contenida en el gráfico $N^{2} 1$, que busca relacionar el curriculum, la praxis docente, la gerencia y la burocracia del sistema educativo, con los principios filosóficos, poli- ticos y sociales que sirven de fundamento histórico-estructural a dicho sistema.

\section{Fundamentos sociológicos de la investigación, la sociedad y el Estado}

La sociedad es un macrosistema social compuesto por una multiplicidad de actores que orientan su acción en base a ciertos principios ideológicos culturales comunes que fueron los fundamentos a partir de los cuales dicha sociedad se constituyó en un momento determinado de la historia. Por su parte, el estado expresa el orden legal y legítimo de una sociedad.

Toda sociedad se rige sobre un acuerdo o imposición entre las clases sociales que la conforman. El acuerdo supone una racionalidad $y$ un orden de los procesos particulares de organización. Así, la sociedad capitalista se levanta sobre la propiedad privada de los medios de producción por parte de la clase burguesa. Clase, que por múltiples procesos se constituye en la clase dominante $\theta$ impohe y orienta un modo de dominación y 
Gráfico No. 1

EL SISTEMA EDUCATIVO Y SU PARADIGMA ORGANIZATIVO

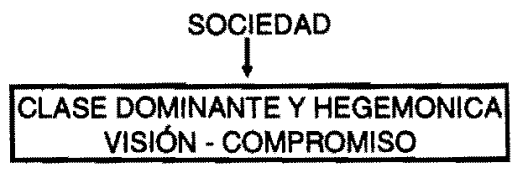

SISTEMA $\mid$ EDUCATIVO

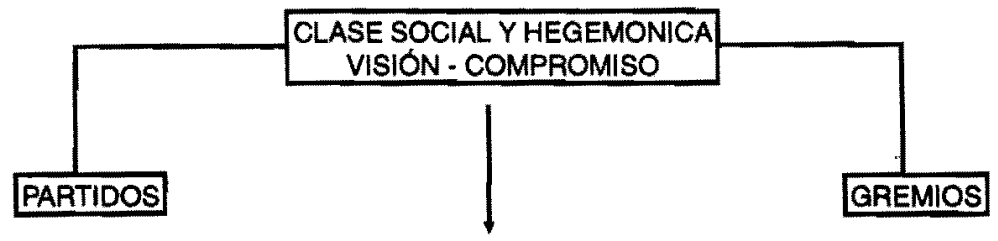

ORGANIZACION

$\downarrow$

GERENCIA

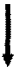

CURAICULUM

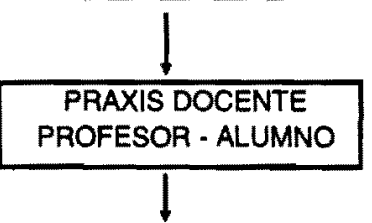

FUNDAMENTOS

EL HOMBRE

ES UN SER

RACIONAL

LA CIENCIA ES LA

UNICA VIA DE

REALIZACION

\section{DEBE EXISTIR UN SOLO ORDEN \\ EN EL MUNDO \\ (LA NO DIVERSIDAD)}

\begin{tabular}{|c|}
\hline EL USO DEL CONTROL \\
PARA MANTENER EL \\
ORDEN O EL ESTADO \\
EXISTENTE
\end{tabular}

ESTRUCTURARLA

SOCIEDAD EN

BASE AL MANDO

Y LA OBEDIENCIA 
legitimidad sobre el resto de las clases y sectores sociales. Las especificidades de cada sociedad se determinan por la manera cómo cada clese social entra a formar parte en la dirección política de esa sociedad, dirección que supone a su vez proyectos históricosparticulares de organización social que van definiendo liderazgos concretos de actuación on los aparatos politicos del sisma social. De este supuesto nace el orden social, manifestado en el estado capitalista, el cual va a tener diferentes formas de gobierno como la dictadura o la democracia. Como podrán observar el concepto de estado alude a un tipo de organización social y un tipo de organización social constituye un paradigma.

\section{Paradlgma soclal}

Por paradigma social vamos a entender a todo modelo de organización social que crea, mantiene y conduce a una sociedad en sus múltiples manifestaciones institucionales. A su vez, todo modelo social contiene los principios filosoficos, politicos, estratégicos y técnicos que orientan el comportamiento de los actores sociales, sean estos clases, fracciones de clase, grupos 0 individualidades. En su origen los actores sociales establecen las reglas de juego a partir de las cuales los mlembros de la Instltución deben asumir y cumplir. LOs acuerdos $8 \theta$ cristalizan en normas generales y particulares, en leyes, reglamentos y procedimientos de acclón social, constituyéndose así el orden político de la Institución. Orden politico particular que a su vez $8 \theta$ conecta y $8 \theta$ relacione con el orden político predominante en la sociedad donde opera la ins- titución. Asi por ejemplo, la LEY ORGANICA DE EDUCACION EN VENEZUELA constituye el orden político $O$ el acuerdo básico que la sociedad admite como legltimo de lo que debería ser la educación.

En definitiva, una norma no es más que la creación de acuerdos entre los actores sociales, de modo que por medio de ella se garantizan los fines de la institución de tal manera que su aceptación y cumplimiento permiten lograr un equilibrio social entre los actores. Pero no solamente las normas viabilizan el equilibrio social, sino que también existe todo un sistema cultural y mental que los actores asumen de modo natural. De alli que el paradigma organizativo que sustenta una institución no solo se capta por medio de las normas, sino también a través de las actitudes, conductas cotidianas y simbolos. El sistema cultural determina el amblente y el clima social de la institución.

Desde la perspectiva histórica todo paradigma social posee un ciclo determinado. Asf tiene un desarrollo, un máximo desarrollo (UMBRAL) y luego entra en una fase de declinación hasta llegar a desaparecer ante la inminencia de otro paradigma social. Los principios ordenadores y los mecanismos para su conservación empiezan a cuestionarse desde el mismo momento en que no responden a las nuevas exigencias sociales.

LOs clclos históricos son dialécticos y no mecánicos, entre una fase y otra pasan afios y hasta siglos para observar cambios significativos.

\section{La crisle social e Institucional}

La crisis corresponde a una forma de significar la declinación del paradigma. 
Al principio la crisis es percibida como un malestar cultural y mental entre los actores; los mecanismos integradores y estabilizadores del sistema no funcionan. Luego aparecen necesidades sociales que no son satisfechas por el sistema social o que históricamente han estado marginadas respecto a otras necesidades sociales, aqui se originan actores sociales que asumen esas necesidades como legitimas y a las cuales hay que satisfacer por la via institucional.

En este momento empieza a aparecer ideológicamente un nuevo paradigma social con nuevos valores y principios. De alli la oposición entre actores y valores. Aparece el conflicto que se aminora o se profundiza según sean los acuerdos - luchas que se liberen en el escenario político y social en general. El conflicto va entonces desde la simple crítica hasta la propuesta de cambio hacia un nuevo modelo social. La crisis exige reformas desde las normas existentes hasta la constitución de nuevas reglas de juego social. La declinación social o entropia social abarca procesos objetivos y subjetivos de participación de los actores sociales, pero solamente habrá cambios cuando las nuevas necesidades sociales son satisfechas en el plano institucional.

En definitiva un paradigma social organizativo es entonces el núcleo o la esencia que sustenta un sistema social determinado. Es en el paradigma y no sus manifestaciones en el sistema social donde hay que buscar las razones de la actual crisis educativa. Por ello, las críticas contenidas van a la estructura y no a las personas que ocupan cargos en las instituciones.

\section{Educación}

Finalmente tenemos el concepto de educación. En su definición general la educación es el proceso por medio del cual la sociedad reproduce y actualiza de manera determinada los valores y principios que contiene el paradigma organizativo que sustenta a la sociedad en un momento de su devenir. En la sociedad moderna se han constituido instituciones expresamente dedicadas a la educación, las cuales se denominan escuelas. Estas instituciones socializan y deben adaptar al individuo conforme a las pautas generales de la sociedad donde se encuentra y conforme a las pautas particulares a la visión de mundo que posee la clase o sector social que dirige la escuela.

La escuela constituye una organización primaria de la sociedad donde al concurrir clases y sectores sociales garantizan la reproducción y/o cambio de una sociedad determinada, de ella emergen las organizaciones secundarias, terciarias o subsidiarias que defiende una fracción o clase en particular, aún por encima de los intereses de la institución primaria.

\section{Los resultados negativos de la Educación}

En Venezuela los resultados del sistema educativo son muy negativos convirtiéndose en un gran fraude tal como lo señalara el actual Ministro de Educación: Es un traude "porque no ha cumplido con las expectativas que el pais habla colocado en ellas" (Cárdenas, 
1995:C.1.). Estos resultados se detectan con algunos indicadores del rendimiento.

En efecto los datos estadisticos señalan que el promedio nacional es de 12.5 puntos con un alto grado de repitencia y deserción escolar. El rendimiento es similar tanto en las escuelas públicas como las privadas. El Ministerio de Educación contabiliza años tras años, de gobierno blanco a gobierno verde cerca de un miIlón de alumnos repitientes y aplazados. Sólo el $14 \%$ de los alumnos terminan la escuela básica y en el caso de la educación superior especificamente en la Universidad del Zulia (LUZ), el $80 \%$ de los estudiantes son irregulares, según información oficial emanada de la Secretarla de la Universidad.

Confirma to anterior, el Dr. Jorge Sánchez Meleán, Presidente de la Comisión para la Relorma del Estado Zulia al señalar que: 1: Actualmente sólo un tercio de los alumnos que ingresan al primer grado logra culminar su educación básica; 2: Un sexto de todos los alumnos que culminan la educación básica tienen conocimientos matemáticos de sólo 5 puntos en una escala de cero a cincuenta; 3 : El sistema escolar no está logrando la contormación de la personalidad de sus egresados en los valores y actitudes que la Constitución y la Ley de Educación establecen; 4: El sistema (educativo) no está capacitando para el trabajo; y 5: EI sistema tiene 180 medios dlas de trabajo escolar en el año, los cuales se reducen al menos a un tercio por los más variados motivos. (Sánchez, 1995:1-4).

\section{La desadaptación como efecto perverso de la educaclón formal}

Paralelamente al bajo rendimiento estudiantil se observa que diariamente se va tejiendo un malestar mental, cultural y social entre los maestros y los alumnos. Malestar que impregna la vida cotidiana de la comunidad escolar. Desde la clase, pasando por la escuela y luego en el hogar se van generando actitudes y conductas de desadaptación social, traduciéndose en los permanentes conflictos personales, grupales, gremiales y politicos de la escuela.

Por un lado, se observa que el alumnos (bien de clase alta, media o baja) no quiere ir a la escuela, la ve como un mundo frustrante y coercitivo. No obstante debe asistir obligado. El nif̌o, el joven $y$ el adulto joven venezolano prefieren otro mundo donde tengan la oportunidad de participar y expresar lo que quieren decir y hacer; donde estén libres de presiones familiares y profesorales. Prefieren el juego, la diversión. Generando entonces conductas de escape mientras que sienten a la escuela como una condena a cumplir durante por lo menos 16 años. Viven como si estuvieran presos o encadenados a una prisión escolar que les resulta extraña a sus intereses y necesidades.

En este escape es cuando caen en las garras del león de la televisión o en los brazos de la pandilla de la droga o el sexo. La T.V. los hipnotiza y lo encanta para que vean como natural la violencia como vía 
para resolver cualquier problema y el sexo como única vía de comunicación con el sexo opuesto. Desde la mañana hasta en la noche, desde los programas para todo público hasta los de censura $C$. Entre un programa y otro, las propagandas se encargan de repetir y repetir el mensaje de la desintegración de la personalidad. En la vida, el alumno sólo aprende lo que el modelo le ha enseñado. Pelea o huye. Se llena de egolsmo o tristeza. Más adelante veremos otras consecuencias de esta situación y la razón que la produce.

La desadaptación termina por traerle secuelas mentales y somáticas como el stress, la psicosis, la neurosis depresiva, la histeria, los dolores de cabeza, la gripe o alergía permanente, los problemas digestivos, circulatorios y nerviosos en general. Tenemos entonces unos efectos bastante fuertes que resul$\tan$ paradojjicos en un estado que proclama en primer lugar, toda una bella filosofía y política de la educación, donde la coloca como una variable estratégica para el desarrollo social de la nación tal como la declara la Ley Orgánica de Educación (1980) en su articulo 4 cuando dice: "La Educación como medio de mejoramiento de la comunidad y factor primordial de desarrollo nacional...". En segundo lugar, concibe la educación como medio para formar al hombre integral en sus dimensiones individuales, sociales y culturales, esto se verifica en la Constitución Nacional (1961) en su artículo 80 que a la letra dice: "La Educación tendrá como finalidad el pleno desarrollo de la personalidad y el logro de un hombre sano, culto y apto para convivir en una sociedad democrática". Esta concepción se amplia en la Ley Orgánica de Educación anteriormente citada en su articulo 3. Además de esta visión el Estado por la vía del Ejecutivo y el Congreso ha realizado una cuantiosa inversión en el sector educativo.

\section{Enfoque causal y holístico de la crisis educativa}

Nos preguntamos ¿qué puede explicar este gran fraude?. Podemos dar respuestas que compilan todo un mundo de variables que en primera instancia $\theta x-$ plican causalmente el problema. Así las causas externas son las siguientes: Las condiciones socioeconómicas de los alumnos unido al alto costo de los útiles escolares; el gobiemo no proporciona el presupuesto necesario y a tiempo para que funcione adecuadamente el sistema educativo; los partidos políticos y los gremios docentes paralizan constantemente las escuelas; los medios de comunicación desnaturalizan la educación; la inestabilidad general de la nación.

Las causas internas son las siguientes: El diseño curricular no se adapta a las necesidades de formación; la escasa o nula motivación para el trabajo docente por parte del profesorado; la mala o pésima pedagogla y didáctica utilizada en el proceso de enseñanzaaprendizaje por parte del docente; la inexistencia de una estructura organizacional adecuada al proceso; la falta de una política de fortalecimiento de los recursos humanos.

Todo este mundo causal de hecho podria explicar por separado $\theta$ inclusive correlacionalmente el bajo rendimiento. 
No obstante ese mundo de variables solo cobrará sentido si se analiza la crisis desde la perspectiva holistica, de manera que puedan vincularse esas causas al paradigma organizativo que precede la dinámica educativa.

\section{La cara brillante y la cara oscura del curriculum escolar}

\section{La pesada carga de la enciclo- pedia}

La filosoffa y politica se materializa solo en la primera parte del curriculum. El resto de las partes de este plan educativo aparece bajo una metodología sistemática de organización del saber pero esconde -como veremos- una forma de entender la educación que termina liquidándola. En otras palabras, en el curriculum se declara en forma brillante ese ideal del hombre integral, pero cuando se estructura dicho curriculum se desnaturaliza y hasta desaparece esa concepción. Expliquemos.

Desde la Revolución Francesa hasta el día de hoy (lqué lamentablej), la estructura curricular de todo el sistema educativo (bésico, medio y superior) es una estructura enciclopedista y atomicista. Se conforma el plan en base a áreas, asignaturas y horas por separado. Se cree (porque al final es una creencia insostenible cientfficamente) que para llevar a efecto el ideal del hombre integral, el alumno debe aprenderse todo to que dice el profesor y el libro enciclopedia que pide oficialmente el Ministerio. Es en esta creencia que empieza a generarse ese gran fraude de la educación no solo a nivel venezolano, sino a nivel mundial.
¿Por qué?, porque la enciclopedia es un sistema de aprendizaje basado en la acumulación de la información. Se cree que la realidad es el libro y que el mundo es el salón de clases y la experiencia es solo el ejercicio de la lectura y comprensión de texto. Para demostrar esto, pongamos una estrategia de evaluación del Programa de estudio del Primer Grado de la Educación Básica. Sector Básica, sector urbano, de Julio de 1985.

El objetivo se considerará logrado cuando el alumno:

- Relacione el fonema (sonido) con sus respectiva grafia.

- Clasifique, en vocales y consonantes un grupo de letras. (Ministerio de Educación. 1985:30).

Bajo esta concepción se confunde el mapa (el texto) con la realidad. El propósito es entonces intelectualista, se cree que educar es saber y transmitir información y esto solamente se comprueba con el examen escrito. En apoyo a esta crítica a los fundamentos de la educación constatamos en el Cuadro $N^{2} 1$ que el alumno debe ver 11 materias en el primer grado, y para pasarlas tiene que aprenderse 58 objetivos generales, 135 objetivos especificos y participar en 290 prácticas de evaluación.

El programa de estudio oficial es semejante a los contratos de los obreros que especifican lo que tiene que hacer desde que entra hasta que termina la jornada de trabajo. La sistematicidad pedagógica pareciera que fuese hecha por los Tayloristas de principio del siglo, que forzaban al trabajador a que fuera eficiente en tiempo y movimiento, y quienes han debido ser buenos alumnos del padre del 


\section{Cuadro 1}

Distribución de las asignaturas de la escuela básica según los objetivos y estrategias de evaluación

\section{Asignaturas}

Objetivos

Objetivos

Estrategias

Generales

Específicos de Evaluación

1. Castellano

2. Matemática

3. Cs. de la Naturaleza

4. Educ. para la Salud

5. Historia de Venezuela

6. Geografía de Venezuela

7. Educación Familiar

8. Estética Educ

9. Estética Artes Plasticas

10. Educación Física

11. Educación para el trabajo TOTAL

11
11
6
5
2
2
4
4
4
6
4

45

63

23

13

10

11

19

22

48

15

21

Fuente: Ministerio de Educación. Programa de Estudio Primer Grado. Educación Básica. Caracas. Editorial Romor C.A. Julio de 1985. (Programa vigente).

conductivismo en Psicología, que supone el aprendizaje como cambios de la conducta provenientes de un estímulo externo.

El programa referido y la concepción que le precede, trunca, en primera instancia, cualquier motivación del alumno, que junto con sus padres se preparan todos los días para acometer la revolución más grande como lo es llegar a ser hombre integral en beneficio de la humanidad, pero que al término de la jornada escolar se ven que han sido traicionados y engañados por los funcionarios contrarrevolucionarios de la pedagogía en la trinchera del centralismo del Ministerio de Educación. Hay en definitiva una visión parcial de la realidad. Realidad que como se demostró solo puede ser abordada mediante áreas-materias, sin conexión alguna, materias sin articulación horizontal o vertical, y contenidos destasados de la cotidianidad del alumno.

\section{La ciencia como único lente del alumno}

Pero la problemática de la enciclopedia se une a la problemática de la ciencia como único valor de sustentación de la vida. Por medio de la ciencia se socializa al alumno para que lea, comprenda y viva la vida de una sola manera. Se inculca al alumno una pretendida racionalidad ajena a todo valor emocional y subjetivo. Se le hace saber al estudiante que la ciencia es un conocimiento que se legitima con la experiencia comprobada, con la evidencia. Por el contrario, aquello que 
no sea evidente es falso e inexistente. (¿Será por eso que Dios no entra en la escuela?). Solo es válido aquello que se puede observar, medir y tocar. Se filtra para siempre, entonces, una concepción utilitarista y pragmática de la vida, vale lo que sea intelectualizado; vale lo que sirve para el individuo y lo que sive es todo aquello que se puede tener, poseer, tocar y ver. La fuente del hedonismo y materialismo se siembran desde el primer grado. Según esta visión de la ciencia, el hombre se realiza por medio del tener: "Soy importante si tengo conocimiento", "Si tengo todos los libros", "Si tengo todos los útiles escolares". Con esto se va fundando también una sociedad basada en una profunda división en clases sociales.

La enciclopedia y la ciencia cumplieron un papel revolucionario cuando se constituyó la sociedad moderna, cuando después de críticar los fundamentos de la sociedad feudal, se recuperó el papel protagónico del hombre frente a las circunstancias, frente a lo inevitable, frente al destino. La ciencia y su acompañante la tecnología se prepararon para construir el nuevo mundo, era la promesa del hombre para alcanzar el desarrollo social. Incluso prohombres modernos elaboraron la gran enciclopedia francesa para demostrar el poderío de la ciencia frente a otro tipo de conocimiento, luego habria que preparar al hombre moderno y para eso nace la escuela; habria que prepararlo para la vida y para que cumpliera un papel activo en la construcción de la nueva sociedad. Entonces fue cuando los maestros de la nueva época tomaron la gran enciclopedia para llenar los contenidos de las diferentes materias y grados. AQUI COMEN-
ZÓ LA TRAGEDIA ESCOLAR. So estructuró el plan de estudios como resultado. Solo como producto que el alumno debería de aprenderse sin cuestiona-miento porque ya los grandes hombres lo habian verificado. No se organizó ni siquiera haciendo referencia al papel político que cumplieron los cientificos, ni la emoción que sintieron cuando descubrieron algo completamente nuevo. A la pedagogía oficial le ocurrió lo que en principio criticaba respecto a la biblia: La biblia se sustituyó por el libro ciencia y el rosario por la repetición de lecciones y más lecciones. Se dogmatizó una concepción de la vida y el saber. Esa concepción desde su origen produjo reacción entre los alumnos generando en éstos la rebeldía, las inhibiciones personales, las fugas masivas, el desorden, la indisciplina escolar, la deserción, la repitencia, la conformación de pandillas de jóvenes, etc.

"Si todo está ya comprobado ¿por qué debo aprendérmelo?" diría el alumno en su conciencia interior. No se toman en cuenta en el salón de clases otras capacidades también intelectuales, como pensar, razonar, discutir, discernir, deducir, porque todo es lección y apuntes, pero lo que es peor, no se toma en cuenta la vida cotidiana del alumno. Aquí no tienen posibilidad de desarrollarse otros valores ante el predominio de la ciencia. No tienen entrada, por ejemplo, el desarrollo natural del juego mientras se es niño, tampoco el desarrollo natural del sexo cuando empieza ese período dificil de la adolescencia.

El alumno debe definitivamente amoldarse a una sociedad que le pide ser alguien en el hacer para que pueda llegar 
a tener, asi le pide desde niño (y los padres lo apoyan) que sea médico, ingeniero, abogado; o si no, debe lograr una educación para el trabajo como carpintero, plomero o herrero. Observamos que aquí se produce el gran circuito que estrella iniciativas y creatividades. La educación se entiende como un proceso que va de afuera hacia adentro (la cabeza del estudiante).

En la confrontación de valores impuestos y necesidades no satisfechas, el alumno busca un escape, pero encuentra, lamentablemente, otras instituciones como la TV, que propician antivalores que inclusive trastocan la ya precaria concepción de la ciencia que se da en la escuela. Esta confrontación le produce al alumno la disonancia mental o una confusión inmensa y depresiva ante la escuela, ante la vida, ante si mismo y en esta confusión queda para siempre neutralizado, sin definir una auténtica personalidad, totalmente dependiente del medio, sin valorarse a si mismo, hedonisticamente preparado, donde el placer por los objetos se convierte en ideal de vida. Asi dice: "Tengo juguetes", o "Tengo los mejores vestidos o zapatos", o "Tengo novia", o "Tengo dinero (en el banco) que me dió mi padre". El resultado es un hombre máquina, un hombre materia, un FRANKENSTEIN, como dijo recientemente Juan Liscano (Liscano, 1995:1-4).

\section{El control social "la cara oculta del curriculum" \\ Pero ¿por qué se impone una for-} ma de hacer ciencia y de ver la vida en la escuela, cuando precisamente la primera se realiza en la verificación como activi- dad permanente?. Para dar respuesta a esto tenemos que traspasar al plano académico para instalarnos en el politico social.

Veamos: La crisis educativa solo se entiende en el contexto de las clases y fracciones de clase que hoy existen. Es una crisis de valores impuesta por una clase frente a otras que no tienen posibilidad de desarrollarse. El desarrollo social en la escuela se va sintiendo como repetición y reforzamiento de un pasado y no de la innovación o avance para el futuro, donde las mayorías pueden participar y beneficiarse.

La clase dominante y IIder como la "burguesla" obtiene su hegemonia y dominación en toda la sociedad y ha logrado con el consenso de la dingencia, que la escuela se estructure en base a una ciencia muerta y una enciclopedia de conocimientos abstractos. Dirigencia que pertenece a la clase media, que con sus organizaciones politicas y gremiales conformaron la sociedad moderna venezolana. Le interesa a aquella reproducir una sociedad que acepte su visión de vida burguesa, pero sin cuestionamiento.

La escuela en ese sentido ha cumplido el papel histórico de mantener el control social de todas las clases sociales subsidianias del sistema mediante mecanismos socializadores para que el control se realice $\sin$ fricciones.

La crisis de la educación no está en relación a la burguesía on sí, ésta se mantiene en la dominación y manda a sus hijos a los centros mundiales del saber para que se formen y hereden su poderío. La crisis está en relación a unas clases sociales como la pequeña burguesía, la 
clase media y los marginales que están siendo excluidas cada dia más del escenario histórico, económico y social. La crisis consiste en que empiezan a aparecer nuevas necesidades sobre las cuales el mundo cultural y social impuesto por la clase dominante, nò da respuesta, respuesta que se dará llegando a un nuevo acuerdo histórico con o sin la clase dominante tradicional.

\section{La crisls de la Educación en Venezuela}

En el caso venezolano la crisis se complica, porque al reproducir la escuela una sola concepción de la ciencia hecha en el extranjero jamás podrá formar a un hombre integral, ni para consolidar una identidad nacional, porque la enciclopedia en su estructura se basa en un conocimiento universal de origen europeo o norteamericano, tampoco permite el fortalecimiento del trabajo, porque el régimen de materias liquida cualquier práctica educativa orientada a la producción, o que conduzca a la generación de capacidades técnicas minimas. La ciencia menosprecia el trabajo manual y otros valores culturales, por ello el ideal de todas las familias es ver a sus hijos en la máxima posición social: ser Doctor. Todo parece indicar una especie de mecanismo histórico politico: La clase dominante impone su modelo de dominación.

En Venezuela en particular, la conformación de un pacto social en 1958, unido al modo de distribución de la renta petrolera, trajo como consecuencia que los sectores en el poder, la burguesla y la clase media perdieran su liderazgo histó- rico no solo en el escenario interno, sino en el internacional al preferir usufructuar la gran riqueza fácil proveniente de la renta petrolera y no activar un programa realmente de desarrollo nacional de corte capitalista, mucho menos de carácter socialista; prefirió aceptar los modelos que otros habian ensayado y aplicado. Esto fue lo que pasó con el comercio al recurrir solo a la importación, así pasó con la industria al no darle impulso y asi paso con la educación al copiar exactamente los modelos y diseños curriculares extranjeros sin adaptación critica y sin experimentar formas concretas en base a nuestras especificidades, tal como lo proponía el maestro del Libertador: Don Simón Rodríguez.

La clase media que ha dirigido el sistema educativo ha carecido de un proyecto politico para si misma, para el sector educativo y para la nación. Copiaron en cada gobierno de turno modelos educacionales y trataron de venderlos como grandes proyectos: "La gran revolución educativa", "La Escuela Básica". Pero fueron eso: modelos copiados que llegaron a reformar lo ya existente, y en el mejor de los casos se pensó innovar la educación solo bajo la forma de la pedagogla, dejando de lado otras dimensiones como la politica, la económica, la administrativa-gerencial, los recursos humanos, etc. Funcionarios tras funcionarios en 37 años de democracia lo que han hecho es mantener el paradigma organizativo heredado e impuesto por la dirigencia politica que ha usufructuado al Estado Venezolano. Todas las innovaciones educativas se han aprobado pero nunca han sido aplicadas por el cuerpo directivo 
comprometido con el modelo de gobierno impuesto. Hoy la crisis de la renta petrolera ha generado expectativas de reforma y cambio, la base de sustentación económica de la burguesía está cuestionada. Las clases medias y pequeña burguesía están totalmente golpeadas. Las marginales y los pobres en general carecen de organización y apoyo. Se ha abierto un periodo de inestabilidad que exige reformular el pacto social para asf poder salir del estado de atraso socio-económico y cultural que tenemos.

El modelo de dominación impuesto explica también, como se verá adelante, el tipo de praxis académica asumida en el aula, el tipo de gerencia escolar predominante y la estructura organizativa implantada.

\section{La praxis educativa o el maestro como obrero de la educación}

Volvamos al mundo académico. La crisis del curriculum se une a la crisis de la praxis educativa dirigida por los maestros y maestras, por los profesores y profesoras. La praxis ha convertido a éstos en simples objetos o simples obreros operarios que usa el sistema para que enseñen lecciones y clases magistrales que la enciclopedia oficial indica, ésta ya viene aprobada por el Ministerio de Educación por medio de un decreto como texto oficial, por grados y también por medio de los programas de las materias. El maestro recibe un programa paquete que contiene un solo orden de objetivos, contenidos, estrategias pedagógicas y estrategias de evaluación, esta práctica es vigilada por el supervisor del Ministerio. La práctica educativa es mecánica y sin sentido, y esto se verifica desde que solo existian maestros graduados en la Normal hasta aquellos que se han graduado en la Universidad o en los centros de mejoramiento de profesional del magisterio. En este contexto -para colmohay quienes señalan que la labor del do. cente puede ser acompañada o sustituida por una computadora.

El maestro tiene la misión de calificar el hombre del futuro, será excelente si el alumno se aprendió todo lo que se le pide, si logra repetir las lecciones impartidas, si contestó todas las preguntas del cuestionario y si hizo todas las tareas asignadas durante los lapsos del año, si no, el alumno no sine y éste tiene que desertar. Un tema tras otro van corriendo durante el año y el niño debe dejar de lado su vida personal para ver si alcanza esas lecciones, pero estas lecciones no le permiten comprender su mundo cotidiano y subjetivo, por ello se explica nuevamente la crisis de la educación.

El alumno no es objeto ni el sujeto de la educación, lo es la ciencia acumulada que impone en forma inmediata la praxis educativa. El maestro se ve como enemigo del alumno, solo califica actividades y no toma en consideración el sentimiento del alumno y es que esa práctica en el aula y esa actitud del maestro tiene, como veremos, un fundamento histórico.

La praxis educativa, en conclusión, solo garantiza el modelo de curriculum adoptado. Las estrategias pedagógicas utilizadas solo abarcan dinámicas formales de educación de los grupos de alumnos, no permiten ni la creatividad ni la 
crítica, además son fraccionadas respecto a los diferentes contenidos curriculares que se ven en forma lineal sin poder volver atrás o de repasarlos cuando se necesitan nuevamente y esto no se hace, porque el alumno es tomado como archivo que debe recordar todo lo que se le dió en forma aislada. Bajo esta concepción el alumno resulta culpable de la crisis, so dice "ahora los alumnos no son como antes".

El maestro en la escuela, como el obrero en la fábrica de tubos, no necesita de ese modelo ni discutir con un equipo, ni horas para el estudlo, $\mathrm{nl}$ necesita la planificaclón estratégica, ni de oficina, ni de tecnologla educativa que facilite el aprendizaje. Es decir, no necesita pensar, por ello es que termina adoptando los medios dlas de los 180 dlas previstos en el calendario escolar, el turno completo no le era indispensable.

Por su parte, el alumno queda entonces reducido al hombre-apuntes, al hombre-loro, Inclusive el régimen de evaIuación continua que se pensó iba a representar un paso decisivo en la educación activa, resulto ser otro mecanismo de castraclón, porque solo sirve para llenar de exámenes a los alumnos y no como mecanismos para mejorar el aprendizaje. El maestro va acumulando y sumando el $70 \%$ y luego toma el examen de lapso con el $30 \%$. Si on esa suma el alumno llega y/o pasa de dlez, entonces ha aprobado el anfo escolar no importando si realmente habrla alcanzado las destrezas minimas como la lectura y la escritura. Los padres tienen que sacar tiempo para haceries las tareas a sus hijos o contratan profesores a domicllo porque el salón de clases no se toma para aprender, sino para escuchar.

\section{La gerencla escolar como vigllancla escolar}

Pero la crisis no solo se nota en el curriculum y en la praxis docente en el aula, sino también en la forma como so conduce la escuela en todo el sistema educativo. Existe una clara separación entre el mundo académico llevado por el profesor en el aula y el mundo administrativo de la escuela y del Ministerio, llevado por los funcionarios. La separación se expllca porque el maestro no está implicado en el proceso de toma de decisiones que ello encierra y que es atribución del aparato administrativo y el cuerpo directivo (jefes, supervisores de sector, distrital, regional o nacional) no están implicados en el proceso académico. La dirección escolar (1) es parcial y se basa, fundamentalmente, en el proceso de control docente, pero el control, cuando se ejer$\mathrm{ce}$, se ve como vigllancia, como hace el policla cuando culda una entrada a un establecimiento comercial. La vigilancia consiste en si se hizo o no la programación del año, si se cumplleron o no las actividades previstas y si el maestro cumple con su jornada de trabajo.

El maestro y la escuela no tienen atribución o competencla gerenclal; es decir, no tienen capacidad de decision académica y administrativa; no planifican porque solo tienen competencia para programar las actividades indicadas on ol plan general del ministerio; no organizan porque todo ya viene normado en cuanto al qué hacer y cómo hacerlo y dónde; se 
les dice quien debe mandar y quien debe ejecutar, no dirigen los recursos estructurales y humanos porque a estos desde que ingresan se les impone qué trabajo han de realizar o qué operaciones estandarizadas deben ejecutar. Los directores no son lideres sino jefes que has-ta ahora son impuestos en su mayorla por la élite politica gobernante en el ministerio, no representan a la colectividad de maestros. Lo único que les queda es supervisar por medio de la vigilancia, cuestión que no incide para nada en la calidad de la educación. Todos los años, de octubre a julio, se repite una rutina escolar sin cambios. El plan de estudios de la escuela básica ya lleva 15 años de vigencia y en la educación superior más de 10 años.

No hay un sistema gerencial cientrfico y humanistico, existe una conducción personal, voluntarista y coyuntural de la educación, no está planteado ser efectivo socialmente. Basta dar el programa sin saber el impacto on el alumno, es suffciente dar la lección sin utilizar los medios didácticos adecuados, basta el pupitre, el famoso pizarrón, la tiza y el borrador. Aquí encontramos el tercer grado de la crisls del sistema educativo. Alumnos, maestros y directivos caen victimas de un modelo educativo, que desde sus fundamentos está mal estructurado y que debemos seguir analizando hasta descubrir su esencia.

La gerencia como ciencia y tecno. logia, no se ha tomado como variable estratégica para el desarrollo educativo, como lo han sido el curriculum y el presupuesto; y es que no se puede tomar en cuenta, porque la gerencia como sistema cientffico busca siempre la readaptación
- cambio de la organización para asl poder lograr su desarrollo espacio temporal, tanto internamente como externamente. A los politicos de la educación al querer el control y la reproducción del modelo social, no les interesa una gerencia innovativa, creativa y autónoma. Al tener control académico, el control politico se da sin dificultad y viceversa, generándose el circulo vicioso de la decadencia escolar. En los palses subdesarrollados y dependientes, el control y la reproducción es más directa al tener a los partidos en las puertas de cada institución escolar, trasladán. dose la toma de decisiones a los burós partidistas y no al consejo de profesores.

\section{El monstruo de la estructura organizativa}

La crisis educativa tiene un cuarto grado de complejidad y tiene que ver con el modelo de estructura organizativa que $8 \theta$ ha asumido. Al nivel fenoménico la estructura es de tipo piramidal vertical. Hay en la cúspide un Ministerio, un Rector, según sea el caso. Los niveles de decisión estratégica se toman a nivel central, mientras que las decisiones tácticas y operativas se toman a nivel medio o de línea. La estructura posee una diversidad de subestructuras especializadas por funclones y actividades. Subestructuras dlrectivas nacionales, regionales y locales, con directores en cada una de las instancias. La organización posee todo un conjunto de normas de funcionamiento y procedimientos que todos deben segulr. Asi nos encontramos con normas sobre cómo diseñar, aprobar y evaluar el curriculum. Hay normas de ingreso, de ascen- 
so y escalafón. Hay normas sobre rendimiento, inscripción, reválida de titulos. Normas y normas que garantizan el fun. cionamiento del gran ministerio, pero que en el fondo lo que garantizan es el control de una élite sobre la mayoría de los docentes.

Pero la crisis de esta estructura social y normativa comienza cuando en la vida diaria no funciona nada en la forma como estaba previsto, todo se tarda; las resoluciones no salen a tiempo. Hay otras prioridades, el nuevo funcionario no le da continuidad a proyectos del gobierno anterior; los nombramientos no se dan; el presupuesto no llega, la nómina estaba mal elaborada, o simplemente "esta emergencia no me corresponde a mi sino a otro funcionario", o "yo no puedo decir nada". En esto hemos detectado más de 10 problemas de funcionamiento, entre otros tenemos: 1) Ruptura de la unidad de mando; se tiene que saltar la cadena de mando porque los jefes intermedios no tienen capacidad de decisión, cadena que es interceptada por los grupos politicos de turno, convirtiéndose en gestores de las necesidades de los subalternos; 2) Ruptura del tramo de control, al estar centralizadas las decisiones, los jefes se copan de funciones y tareas violentando la jerarquia de mando existente a nivel intermedio, quedando éstos como simples supervisores o mensajeros de los de jerarqula superior; 3) Una cadena de mando excesivamente larga, impidiendo el flujo de información vertical descendiente y ascendiente, impide también tomar las decisiones a tiempo, dado el divorcio entre funcionario que está en la cúspide y el maestro disperso $\theta$ incomu- nicado de las escuelas urbanas y rurales; 4) La constitución de una diversidad de estructuras organizativas, muchas de ellas en paralelo, que en vez de facilitar el proceso gerencial lo que hacen es complicarlo, generando ambigüedad $\theta$ indetinición de poderes. Se intenta delegar pero a nivel de tareas y responsabilidades, no en decisiones; la descentralización del sistema educativo se ha hecho solo en la parte operacional y no a nivel estratégico. Siguen mandando los mismos, sin solucionar en concreto ningún problema académico o gremial; 5) Una concentración en la cúspide del poder académico y politico, desmotivando a los directores y maestros que diariamente son los que viven los problemas de la escuela; 6) La aparición de organismos informales como los gremios, que trente a un incumplimiento del contrato colectivo paralizan todo el sistema educativo nacional y regional.

\section{La racionalidad burocrática: el núcleo de la crlsls}

La pirámide educativa posee una esencia paradigmática que al descubrirla termina explicando en su totalidad la crisis educativa. La esencia de esta organización es la burocracia, la forma más racional de organización social, donde se verifica "una acción racional (objetiva y fria) con arreglo a determinados fines" (Weber, 1974:179-181) (2). Esta racionalidad es la que define la sociedad moderna, la cual se fundamentó en la creencia que el hombre es un ser absolutamente lógico, capaz de llevar hasta sus últimas consecuencias su rezón para ordenar y 
sistematizar su vida, de all la razón cientifica impuesta en el curriculum, de allí la razón normativa impuesta en los sistemas sociales; pero en el fondo de esto, dicha racionalidad es para dominar; para no desviarse, el hombre debe normarle a otros hombres o sujetos su comportamiento. Los padres a los hijos, los jeles a los subalternos. Debe normarse lo que se tiene que saber y conocer (las capacidades), para asi poder cumplir a su turno un papel en la división social y técnica del trabajo en todas y cada una de las instituciones de la "SOCIEDAD", sean éstas: empresas, escuelas o dependencias del gobierno.

Lo anterior tiene un trasfondo polltico y es que el sujeto histórico que asumió y desarrolló esta racionalidad, como lo fue la burguesfa, la convirtió en el único eje de la vida social, por ello es que todos los individuos tenfan que adaptarse de una forma u otra a este modo de vida. Aparece entonces el control social porque no se aceptó históricamente la diversidad social como base de la convivencia humana, sino como base de la estratificación del poder. De modo que la otra cara de la razón cientffica es el control unido a la lógica mando-obediencia. Y este trípode es la base de todas las organizaciones clviles y políticas de la sociedad modema.

Esta lógica racionalista es la que asume la escuela en su organización, en su praxis educativa y on su curriculum. En conclusión, hemos armado el rompecabezas de esta crisis que no se debe ni a directores, ni a maestros, ni a los estudiantes; es el paradigma organizativo que sine de sustancia a la sociedad actual. Ampliemos el análisis. La racionalidad ex. plica entonces por qué la centralización del poder desde el Ministerio de Educación; explica el por qué del autoritarismo. donde unos deciden por otros, donde se hace uso de la ley o de la fuerza para dominar a otros y donde los demás deben ejecutar como policías u obreros la ley impuesta por el grupo de mando. Estos supuestos están en la presentación del programa oficial de la escuela básica que ya hemos citado anteriormente. Aquil leomos esa lógica de mando:

\section{-... La Oficina Sectorial de Planificactón y Presupuesto realizo la evaluación de los diseños curriculares de la Escuela Primarta y del Clelo Báslco Común y del onsayo de Educaclón Básica a fin de PROCEDER conjuntamente con la DF- rección General de Docencia, a ELA. BORAR LOS NUEVOS DISEÑOS...: (Ministerio de Educación, 1985:2).}

La racionalidad burocrática consume y consume toda la energía del mundo de los funcionarios, pero nunca resuelve nada de modo significativo. Los problemas vuelven y vuelven a aparecer, ministros tras ministros se esfuerzan por superar la problemática, pero mantlenen Intactos los supuestos de esta racionalidad. Ante una supervisión que no funciona proponen formar "los verdaderos supenisores"; ante un curriculum que no sive para que aprendan a leer y escribir proponen "diseñar unos que cientificamente si lo logren"; ante un maestro que no está capacitado, plantean "que debe estarlo". Pero ésta no es la vía de solución, porque se mantiene el burócrata, las nomas y los 
procedimientos que impulsaron precisamente esta crisis.

La racionalidad burocrática sólo sirve para el control, pero no para el desarrollo académico, mucho menos para el desarrollo del hombre. Este aparece siempre como status dentro de la estructura social y no como un ser que posee problemas y necesidades diversas, con racionalidad y emotividad, con aspiraciones $\theta$ ideales distintos a los que mandan (3). El maestro ha vivido prácticamente en la jaula de hierro de la burocracia escolar. Pero a estas caracteristicas personales se une la crisis de una sociedad inestable y un gobierno que ha perdido la legitimidad y ante esto se busca un cambio que permita el desarrollo humano de los alumnos y profesores, maestros, directivos y representantes. Se busca ahora una nueva racionalidad donde los autores puedan autodeterminarse, donde sea posible la coparticipación en la toma de decisiones y en las tareas diarias, donde exista una jerarquía pero no basada en el control, sino en la integración y en la eficiencia, para así lograr la satisfacción de las nocesidades y aspiraciones de la comunidad educativa; por ello fue un acierto la Convenclón de Gobernadores realizada en la cludad de Maracaibo cuando declararon la necesidad de constitulr el municiplo escolar, donde el plantel sea el centro de la gestión educativa. Igualmente es un avance el contenido del IX Plan de la Nación en materia educativa, porque busca una nueva dirección del proceso, al afirmar que "la descentralización del sistema escolar, en cuanto estrategia, debe acentuar el carácter de la educación como proceso de interacción constructi- va. Esa estrategia debe diseñarse desde la escuela como comunidad...". (CORDIPLAN, 1995:186).

El tracaso de las retormas educativas ha sido precisamente porque se ha visto con la óptica burocrática y populista donde a los alumnos y a las comunidades escolares hay que darles todo lo que necesitan y éstos, por no tener un ideal -señalan- hay que ayudarlos; pero se ha dado para que reproduzcan un estado social determinado. Hoy por el contrario, la reforma 0 el cambio educativo debe centrarse en la ciencia como proceso de construcción unido a otras formas culturales como el arte, la religión, la filosofla, donde se puedan compartir valores que fortalezcan y ayuden a construir una identidad nacional. Se debe mejorar la calidad de vida del maestro. No tenerlo como un cordero, sujeto al mecate del hambre y a la cadena de los bajos salarios, de lo contrario van a aparecer de nuevo los sindicatos y gremios, paralizando el sistema.

Hoy hay semillas sembradas tanto en los departamentos marginales del Ministerio como en las diferentes comunidades, que ya empiezan a germinar y que representan el nuevo paradigma social. Asi tenemos a los programas sociales y de salud, el Maestro Promotor de la Salud, el programa Fe y Alegrla, el Programa del Centro de Educación Popular (CEP) y el Programa de Valores Humanos, entre otros. Estamos en los albores de una nueva era que algunos han denominado post-modernidad.

\section{Conclusiones}

La crisis del sistema educativo no 
se debe a la crisis de gestión, ni a la excesiva ingerencia de los gremios, ni a la falta de motivación y responsabilidad de maestros.

Todo esto es la fenomenologla del sistema social y educativo. Representan los efectos, los sintomas del problema. Se debe en verdad a la crisis de los supuestos filosóficos y polfticos que definieron la aparición de la sociedad moderna. Supuestos que conducen solo al control social, donde se ve el hombre en un sentido pragmático como funcionario o como un ser con la capacidad para ejercer un puesto en el concierto de la división social del trabajo.

El curriculum desde la primaria a la Universidad, presenta una franca contradicción entre la necesidad de formar al hombre integral y una estructura curricular que lo único que garantiza es la reproducción de un orden social basado en el intelectualismo, la especialización, en el libro ya elaborado y en el control social. La praxis educativa se reduce a una práctica mecanicista y rutinaria que impide la creatividad del maestro en el aula y en la escuela.

La praxis gerencial es rutinaria y no es sistemática, la cual genera una desarticulación entre el mundo docente y las funciones del aparato administrativo.

La organización piramidal pensada para que el sistema marche racionalmente, resulta infuncional porque no resuelve a tiempo los problemas y necesidades de las escuelas. La burocracia es incompatible con el ideal educativo de la nación, porque deja de lado la potencialidad del maestro y lo reduce a un funcionario que debe aplicar y obedecer normas y planes previamente elaborados por la élite aobernante.

Lo anterior plantea la necesidad de que los maestros y directores, asI como los padres y representantes de todos los niveles educativos, definan los nuevos valores que garanticen un verdadero desarrollo nacional y humano en la educación. También plantea la necesidad de ensayar nuevos modelos curriculares que permitan viabilizar los nuevos valores. Los planes de estudio deben concebirse como procesos ablertos al desarrollo de la personalidad de los alumnos, maestros y comunidad en general, se recomienda tomar como centro del curriculum, las necesidades de los alumnos se. gún sea el medio social y las condiciones en donde se encuentran. Por ejemplo, el Programa Maestro Promotor de la Salud para las escuelas de alta marginalidad.

Es necesario ensayar formas de organización escolar descentralizadas con un alto sentido del compromiso y participación social. En tal sentido, se propone un modelo de transición donde por un lapso de un año el poder reglonal del Ministerlo sea transferido a las parroquias. En este año traspasar el poder académico a las escueles, previa capacitación de los particlpantes. Convertir la estructura burocrática existente en redes parroquiales, regionales y nacionales de integración y apoyo educativo.

Igualmente, es necesario disenar y aplicar un plan de desarrollo del maestro y del directivo, a los efectos de lograr una estabilidad laboral y social, de manera que sean realmente los actores del cambio educativo nacional.

En tal sentido se pone a disposición 
el Programa de Gerencia Académica de la Facultad de Ciencias Económicas y Sociales de la Universidad del Zulia.

\section{Notas}

(1) Para los fines de'este trabajo se han utilizado de manera análoga los términos dirección escolar, gerencla educativa y administraclón educativa. Sin embargo, desde el punto de vista técnico y según los marcos téricos de referencia, se pueden hacer distinciones. Véase al respecto: Ochoa H., Haydeo y Chirinos, Emilio, "Administración y Gerencia, ¿fenómenos diferentes?. Una discusión necesaria". Maracaibo. Facultad de Ciencias Económicas y Sociales de LUZ. 1996. 18 págs. (Mimeografiado).

(2) La burocracia como fenómeno histórico social ha sido abordada desde diferentes posiciones que revelan su pro y su contra. Al respecto remitimos al lector al excelente trabajo de Kliksberg, Bernardo, "El Pensamiento Organizativo. De los Dogmas a un nuevo Paradigma Gerencial". Argentina. Editorial Tesis, 1990. Véase también: Kliksberg, Bemardo (Compilador), "Cuestionando en Administración". Buenos Aires, Argentina. Editorial Paidos, 1973. (3) Para un análisis más detallado véase: Méndez Q., Evaristo. "Gerencia Acadómica". Maracaibo. EDILUZ, 1995.

\section{Referenclas Blbllográflcas}

Asociación de Cobemadores de Venezuela.
Declaración del Zulla. En el Diario El Nacional. Domingo 25 de Junio de 1995.

Cárdenas, Antonio Luis. La Educación es un Gran Fraude". Diario El Nacional. 6-2* 95.

CORDIPLAN. IX Plan de la Naclón. 1995.

Congreso de la República de Venezuela. Constitución Nacional. Gaceta Oficial 1961.

Congreso de la República de Venezuela. Ley Orgánica de Educación. Gaceta Oflcial N $N^{2} 2635$ Extraordinario del 28 de Julio de 1980.

Kliksberg, Bernardo. El Pensamlento Organlzativo. De los Dogmas a un nuevo Paradlgma Gerenclal. Argentina. Edltorial Tesis. 1990. 457 pag.

Kllksberg, Bernardo (Compllador). Cuestlonando en Administración. Buenos Aires, Argentina. Editorial Paidos. 1973. 211 pag.

Liscano, Juan. Blenvenldos al cyber-paralso. En el Diario El Nacional. Junio de 1995.

Ministerio de Educación. Memorla y Cuenta Varlos Años.

Ministerio de Educación. Programa de Estudio. Primer Grado Educación Básica Sector Urbano. Editorial Romor C.A. Caracas, Jullo de 1985. 432 pag.

Méndez Quintero, Evaristo. Gerencla Acadómica. Maracalbo. EDILUZ. 1995. 309 pág.

Ochoa, Haydee y Chirinos, Emillo. Administraclón y Gerencla ¿tenómenos diferentes?. Maracaibo. Facultad de Cienclas Económicas y Sociales. 1996. 18 pág. (Mimeografiado).

Weber, Max. Economla y Socledad. México. Editorial Fondo de Cultura Económica. 1974. Tomo I. 665 pág. 